\title{
Modelagem da distribuição geográfica de espécies lenhosas de cerrado no Estado de São Paulo
}

\author{
MARINEZ FERREIRA DE SIQUEIRA ${ }^{1,3}$ e GISELDA DURIGAN²
}

(recebido: 02 de junho de 2005; aceito: 12 de abril de 2007)

\begin{abstract}
Modeling geographic distribution of "cerrado" woddy species in São Paulo State). Many different techniques are used today with different objectives to model species geographic distribution. Some of these techniques are based on environmental analysis where algorithms search for environmental conditions similar to those where the species are known to occur, resulting in potential areas where the environmental conditions would be favorable for the growth of these species. The present study is based on predictive modeling of species geographic distribution using genetic algorithm as a tool for plant conservation in modeling the "cerrado" biome of the State of São Paulo. The methodology used and the results obtained were considered satisfactory for the generation of geographic distribution models for plant species, based on abiotic data for the regions studied. The accuracy of the model in predicting the occurrence of species in the "cerrado" is greater if only points that occur within the "cerrado" and not in transition areas are used. In order to minimize problems due to the lack of convergence of the genetic algorithm GARP (“Genetic Algorithm for Rule Set Production”), 100 models were generated for each specie. The use of modeling tools can help understand the distribution patterns of a biome or ecosystem in a regional analysis.
\end{abstract}

Key words - biodiversity modeling, genetic algorithms, plant species

RESUMO - (Modelagem da distribuição geográfica de espécies lenhosas de cerrado no Estado de São Paulo). Atualmente vêm sendo desenvolvidas e utilizadas várias técnicas de modelagem de distribuição geográfica de espécies com os mais variados objetivos. Algumas dessas técnicas envolvem modelagem baseada em análise ambiental, nas quais os algoritmos procuram por condições ambientais semelhantes àquelas onde as espécies foram encontradas, resultando em áreas potenciais onde as condições ambientais seriam propícias ao desenvolvimento dessas espécies. O presente estudo trata do uso da modelagem preditiva de distribuição geográfica de espécies nativas, através da utilização de algoritmo genético, como ferramenta para auxiliar o entendimento dos padrões de distribuição do bioma cerrado no Estado de São Paulo. A metodologia empregada e os resultados obtidos foram considerados satisfatórios para a geração de modelos de distribuição geográfica de espécies vegetais, baseados em dados abióticos, para as regiões de estudo. A eficácia do modelo em predizer a ocorrência de espécies do cerrado é maior se forem utilizados apenas pontos de amostragem com fisionomias de cerrado, excluindo-se áreas de transição. Para minimizar problemas decorrentes da falta de convergência do algoritmo utilizado GARP ("Genetic Algorithm for Rule Set Production"), foram gerados 100 modelos para cada espécie modelada. O uso de modelagem pode auxiliar no entendimento dos padrões de distribuição de um bioma ou ecossistema em uma análise regional.

Palavras chave - algoritmos genéticos, espécies vegetais, modelagem de biodiversidade

\section{Introdução}

Originalmente o Estado de São Paulo apresentava uma cobertura florestal correspondente a $81,8 \%$ do seu território (Victor 1975). Do ponto de vista de área total remanescente, a situação do cerrado (sentido lato) no Estado é ainda mais devastadora do que a da Mata Atlântica. O bioma cerrado, em São Paulo, abrangia originalmente um quinto do Estado (Eiten 1970). Porém, naquela época, alguns autores comentaram a inadequação dos mapas de vegetação em seus trabalhos e experiências

\footnotetext{
1. Centro de Referência em Informação Ambiental - CRIA. Av. Romeu Tórtima 388, Barão Geraldo, 13083-855 Campinas, SP, Brasil.

2. Instituto Florestal de São Paulo - IF. Horto Florestal de Assis. Caixa Postal 104, 19800-000 Assis, SP, Brasil.

3. Autor para correspondência: marinez@cria.org.br
}

de campo. Eiten (1972) discute essas limitações, segundo o autor nenhum mapa delimitava satisfatoriamente a área de cerrado no Brasil, especialmente em relação às áreas limítrofes, de contato com outros tipos de vegetação e isso era particularmente evidente no Estado de São Paulo. Ainda segundo Eiten (1972), o melhor mapeamento da época, apesar de excluir algumas áreas, foi o de Borgonovi \& Chiarini (1965) que, no início da década de 1960 , realizaram um levantamento do cerrado a partir de fotografias aéreas de 1962 . O resultado deste levantamento apresenta a área de cerrado ocupando cerca de $15,4 \%$ do território paulista. No começo da década de 1970, esta área já estava reduzida em cerca de 4,18\%, (Serra Filho et al. 1974). Atualmente esta área é de menos de $1 \%$ da sua distribuição original (Kronka et al. 1998), cobrindo apenas cerca de 23.790 ha, de forma extremamente fragmentada. 
Essa situação se agravou ainda mais quando o cerrado foi excluído do decreto 750 (IBAMA 1993), que dispõe sobre o corte, a exploração e a supressão de vegetação primária ou nos estágios avançado e médio de regeneração de Mata Atlântica. Por conseqüência, a devastação do cerrado no Estado atingiu níveis altíssimos. A velocidade da devastação levou à realização do "workshop" "Bases para conservação e uso sustentável das áreas de cerrado do Estado de São Paulo" (SMA-SP 1997), com o objetivo de realizar um diagnóstico da situação atual das áreas de cerrado no Estado e propor diretrizes para a sua conservação.

As áreas prioritárias apontadas neste "workshop" foram alvo de estudo pelo projeto "Viabilidade de conservação dos remanescentes de cerrado do Estado de São Paulo" dentro do Programa Biota (BIOTA 1999) e os resultados do levantamento de flora deste projeto encontram-se disponíveis no site do SinBiota (SinBiota 1999). Estes dados serviram de base para a publicação recente de alguns artigos sobre padrões fitogeográficos do cerrado paulista. Durigan et al. (2003a) analisaram a flora arbórea de 202 remanescentes de cerrado ocorrentes em São Paulo e em estados vizinhos. Neste trabalho, a distribuição das áreas geradas por análise multivariada sugere que as relações de similaridade se estabelecem em primeiro lugar em função do clima e em segundo lugar, em função da fertilidade dos solos.

A vegetação de cerrado é decorrente de vários fatores, sendo os mais importantes: estação seca definida, fertilidade e drenagem do solo, regime de fogo, e as flutuações climáticas do Quaternário (Oliveira Filho $\&$ Ratter 2002). Dentre esses, o solo é hoje considerado um dos fatores mais importantes para a distribuição de espécies e a variação florística no cerrado (Ratter \& Dargie 1992).

As principais classes de solos na região dos cerrados são os latossolos podzólicos, cambissolos, areias quartzosas, litólicos, laterita hidromórfica e gleis. Esses tipos de solos apresentam baixa capacidade de armazenamento de água, ou seja, são bastante lixiviados $\mathrm{e}$, conseqüentemente, possuem baixa fertilidade natural, acidez elevada e altos níveis de alumínio trocável (Vargas \& Hungria 1997).

Em termos geomorfológicos, a região Central do Brasil é um exemplo clássico de evolução policíclica da paisagem, ou seja, é constituída de formas jovens de superfícies oriundas do Pleistoceno e remanescentes de superfícies muito mais antigas (Motta et al. 2002).

Vários autores analisaram as questões relativas às condições climáticas e à vegetação durante o período
Quaternário no Brasil Central (Behling 1998, Behling 2003, Ledru et al. 1998, Salgado-Labouriau et al. 1997, Salgado-Labouriau 1998, Salgado-Labouriau et al. 1998). Estes estudos envolvendo o passado têm confirmado que as florestas e o cerrado mostraram sucessivas expansões e retrações, seguindo as flutuações climáticas do Quaternário (Ledru 2002).

Segundo Behling (1998), a área de cerrado no Sudeste do Brasil foi bem maior no começo do Holoceno (cerca de 10.000 anos A.P. - Antes do Presente) do que no final deste período. As ilhas de cerrado encontradas hoje no meio da floresta estacional parecem indicar uma distribuição anterior do cerrado bem maior. Mais especificamente, há cerca de 32.000 anos A.P., o clima era quente e úmido. Os registros mais antigos de pólen de cerrado são dessa época (Ledru 2002). De 30.000 a 26.000 A.P. o clima tornou-se mais frio, mas ainda úmido. De 19.000 a 7.000 anos A.P. houve uma época mais seca, com a presença de desertificação em várias áreas, com predomínio de formações de cerrado aberto. Cerca de 7.000 anos A.P. a umidade começou a aumentar, o que levou a uma expansão das florestas de galeria e da floresta estacional. De 4.000 anos A.P. até o presente o cerrado arbóreo e a floresta estacional chegaram aos níveis atuais de abrangência (Ledru et al. 1998). Os encraves de cerrado presentes hoje na Amazônia e em São Paulo podem ser resultantes destas expansões do passado (Ledru 2002).

Para algumas áreas a vegetação de cerrado foi substituída pela floresta semidecidual, mas isso somente no último milênio, sugerindo que o fim do Holoceno foi um período mais úmido (Behling 2003). Segundo o autor, por volta de 7.560 anos A.P. as florestas de galeria se expandiram nos vales, demonstrando uma maior umidade. Porém, entre 7.560 e 6.000 anos A.P., houve um retorno às condições mais secas e a savana voltou a se expandir, mas entre 6.000 e 2.180 anos A.P., os vales foram novamente cobertos por florestas semideciduais, mas nas regiões mais elevadas o cerrado aberto continuou prevalecendo. Essa situação mudou entre 2.180 a 600 anos e a vegetação de cerrado aberto nos topos mudou para cerrado mais fechado, refletindo as condições mais úmidas do período. E finalmente, apenas há 600 anos, a floresta semidecídua vem se expandindo nesta região (Behling 2003).

Contudo, desde então, apesar dos níveis de umidade terem aumentado, as florestas não retomaram sua distribuição original em relação ao cerrado. Isso parece estar ligado a fatores como a utilização de fogo, cujo uso recorrente, assim como o empobrecimento do solo, associado a períodos de seca, tendem a impedir a 
sucessão de espécies florestais (Oliveira-Filho \& Ratter 2002). A distribuição atual do cerrado e da floresta estacional no Estado de São Paulo apresenta um padrão de mosaico bastante evidente, sendo impossível, muitas vezes, determinar onde uma formação termina e a outra começa.

Visando a fortalecer estratégias de conservação, diante do atual estado de fragmentação deste bioma no Estado de São Paulo, foram aplicadas neste estudo técnicas de análise espacial e ferramentas de modelagem que pudessem predizer a potencial distribuição do bioma cerrado no Estado, baseadas em parâmetros ambientais como o clima e o meio físico do presente. Com base em dados biológicos (pontos de ocorrência de espécies) e dados ambientais (mapas temáticos), foram aplicados algoritmos com o objetivo de modelar o nicho fundamental e a área potencial de ocorrência dessas espécies.

Neste trabalho pretende-se responder às seguintes perguntas:

É possível usar modelagem para mapear as áreas potenciais de distribuição atual do cerrado no Estado de São Paulo?

A mistura de diferentes biomas na modelagem afeta a eficácia do modelo em predizer os pontos de teste?

Para o conjunto de dados disponível, qual o grau de incerteza que a falta de convergência do algoritmo genético acarreta nos modelos resultantes?

\section{Material e métodos}

Para a realização deste estudo, foram amostradas 86 áreas, originalmente mapeadas como cerrado (Kronka et al. 1998), localizadas em 40 municípios do Estado, tendo sido registradas 548 espécies lenhosas, das quais 17 foram selecionadas para a realização de modelagem. O levantamento dessas 86 áreas foi realizado dentro do escopo do projeto "Viabilidade de conservação dos remanescentes de cerrado do Estado de São Paulo", cujo objetivo foi realizar um levantamento da flora e do estado de conservação dos remanescentes, em áreas não protegidas por unidades de conservação, dentro do Estado. Adotou-se, para o levantamento da flora neste projeto, um método de inventário florístico expedito, no qual a suficiência de amostragem baseia-se em uma curva de espécies $\mathrm{x}$ tempo. $\mathrm{O}$ método, inicialmente utilizado por Ratter et al. (2000), encontra-se descrito em Durigan et al. (2003b), que apresentam a análise dos padrões fitogeográficos encontrados para o cerrado em São Paulo, resultado do referido projeto.

As análises desenvolvidas neste artigo tratam da distribuição do cerrado no Estado de São Paulo. São apresentados na figura 1 os tipos de vegetação ocorrentes no Estado, assim como as áreas de contato entre os diferentes tipos segundo o IBGE (1988) e as localidades amostradas no projeto.

Escolha das espécies - Para a realização da modelagem foram escolhidas 17 espécies (tabela 1) por apresentarem os maiores conjuntos de pontos disponíveis, ou seja, registradas em 20 ou mais localidades diferentes dentre as 86 áreas levantadas e por serem espécies características do cerrado de acordo com Durigan et al. (2004). A escolha deste limite de corte (20 localidades) seguiu as recomendações para o algoritmo utilizado GARP - "Genetic Algorithm for Rule Set Production" (Stockwell \& Peterson 2002) para se conseguir um bom resultado com a modelagem.

Embora sejam típicas de cerrado, essas espécies são eventualmente registradas em áreas ecotonais ou de contato entre o cerrado e a floresta estacional semidecidual (Durigan et al. 2004).

Parâmetros ambientais utilizados para a modelagem - Dados climáticos - Worldclim version 1.1 Global Cimate Surface (Hijmans et al. 2005). Resolução 0.83 km. Precipitação mensal (12 camadas); temperaturas máximas mensais (12 camadas); temperaturas médias mensais (12 camadas); temperatura mínimas mensais (12 camadas). Dados topográficos -U.S. Geological Surveys (EROS 1996). Resolução de 1 km: elevação (uma camada); relevo (uma camada); aspecto do terreno (uma camada); capacidade de retenção de umidade (uma camada). Mapa de solos do Estado de São Paulo - Instituto Agronômico de Campinas. Escala 1:500.000 (uma camada) (Oliveira et al. 1999). O mapeamento pedológico apresenta os dados categorizados e não contínuos. Quando os dados são divididos em classes, os valores originais são perdidos, o que para efeito de modelagem é bastante limitante, mas, devido à grande importância dos solos na distribuição de espécies vegetais, esta variável foi incluída nas análises de modelagem deste estudo.

Método de Modelagem - Neste trabalho foi usado o algoritmo genético GARP (Stockwell \& Noble 1992). Este algoritmo permite prever a distribuição geográfica potencial a partir de modelos de nicho fundamental de espécies. Os resultados da modelagem foram visualizados e analisados através de um Sistema de Informação Geográfica (ArcView 8.2 - ESRI).

Para efeito de comparação da eficácia do modelo em predizer áreas de cerrado no Estado de São Paulo, foram realizados dois experimentos.

Experimento A: modelagem utilizando apenas pontos de ocorrência das espécies de cerrado, em fragmentos de cerrado.

Experimento B: modelagem utilizando pontos de ocorrência das mesmas espécies de cerrado presentes também em áreas ecotonais e bordas de áreas florestais, além das áreas de cerrado.

Para realizar os experimentos, os conjuntos de dados biológicos disponíveis foram divididos em $50 \%$ para pontos de treino (tabela 1) e 50\% para pontos de teste para cada 
espécie modelada. Os pontos de teste são utilizados apenas para calcular a porcentagem de acerto de cada espécie, não participando, portanto, da geração do modelo.

Este teste é feito através da comparação célula a célula entre a matriz resultante da soma de modelos e a planilha de dados de campo para a área de estudo. Esta comparação mostra o quanto o modelo acertou ou errou na previsão. A contagem é feita a partir dos números de teste da seguinte forma:

Verdadeiro: quando o modelo e a planilha de campo dizem que a espécie ocorre ou quando ambas dizem que não ocorre.
Falso: quando o modelo diz que a espécie ocorre e a planilha de campo diz não, ou vice-versa.

N/A: quando o campo diz que a espécie ocorre, mas trata-se de um ponto de treino (ou seja, utilizado na modelagem). Neste caso este dado não é contabilizado, não se aplica.

A porcentagem de acerto dá uma idéia de quanto o modelo é capaz de acertar a previsão de ocorrência (presença) ou de não ocorrência (ausência) para cada espécie. Considerando que todas as espécies utilizadas são bastante abundantes, ou seja, ocorrem com vários indivíduos no

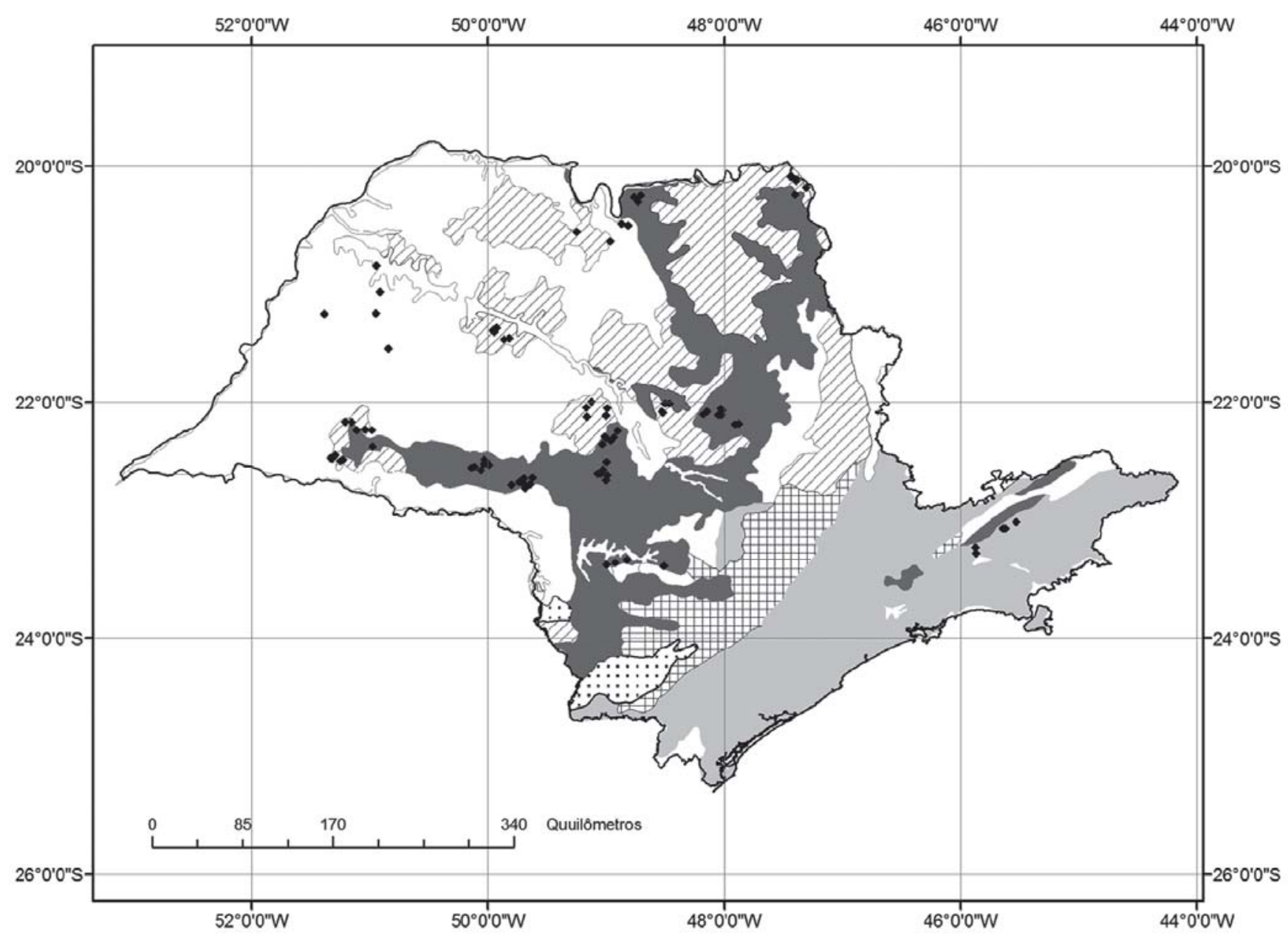

Fıgura 1. Mapa da vegetaçao do Estado de Sao Paulo edıtado a partır do Mapa de vegetaçao do Brasıl, digıtalızado por U.S. Geological Survey's (USGS) EROS Data Center, Sioux Falls, South Dakota (IBGE 1988) com as localidades amostradas no projeto "Viabilidade de conservação dos remanescentes de cerrado do Estado de São Paulo". $\diamond=86$ localidades amostradas; $\square=$ Floresta Ombrófila Densa; $\square=$ Floresta Estacional Semidecidual; $::::=$ = Floresta Ombrófila Mista; $\square=$ Cerrado; WIIA = Contato Cerrado - Floresta Estacional Semidecidual; 曲曲 = Contato Cerrado - Floresta Ombrófila Densa.

Figure 1. Vegetation Map of São Paulo State, modified from Vegetation Map of Brazilian Vegetation, digitalized by U.S. Geological Survey’s (USGS) EROS Data Center, Sioux Falls, South Dakota (IBGE 1988) with the records sampled by the project "Viabilidade de conservação dos remanescentes de cerrado do Estado de São Paulo". $\diamond=86$ sampled localities; $\square=$ Atlantic Forest; $\square=$ Atlantic Semi-deciduous Forest; $::::$ = Altitude Atlantic Forest; $\square=$ "Cerrado"; $V I I / A=$ Transition betwen "Cerrado" and Atlantic Semi-deciduous Forest; 姍曲 = Transition between "Cerrado" and Atlantic Forest. 


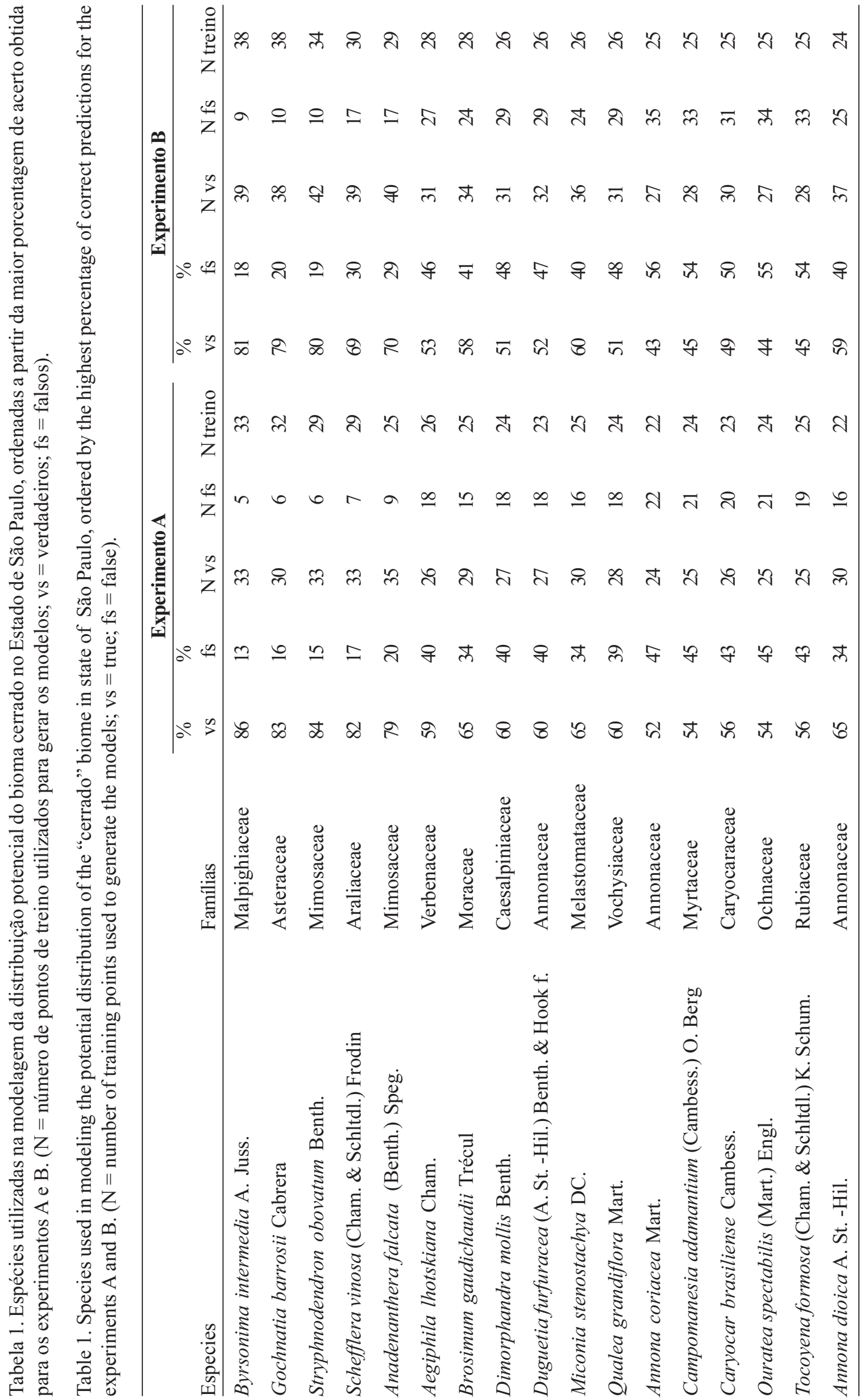


campo, foi considerada ausência da espécie quando nenhum indivíduo da espécie foi encontrado no fragmento aplicandose o método expedito de levantamento botânico.

Para um melhor resultado são gerados vários modelos. No presente estudo foram gerados 100 modelos por espécie, dos quais foram escolhidos os dez melhores modelos (Anderson et al. 2003) que, somados (sobrepostos utilizandose Sistema de Informação Geográfica - ArcView 8.2 - ESRI), compuseram o modelo de distribuição potencial de cada espécie. Posteriormente os modelos gerados para cada espécie foram somados para produzir os mapas com a distribuição potencial das áreas de cerrado.

Uma grande crítica ao uso de algoritmos genéticos é a falta de convergência dos resultados gerados, ou seja, cada vez que o algoritmo é gerado, com o mesmo conjunto de dados, o resultado pode ser diferente. Para saber se ocorreu esse efeito, neste conjunto de dados, foi realizado um teste, a partir dos pontos de treino de Byrsonima intermedia A. Juss., para os mesmos parâmetros ambientais. A questão aqui é saber se a falta de convergência na repetição de um mesmo experimento, que é uma característica intrínseca de algoritmos genéticos, produz realmente modelos muito distantes (não semelhantes) para o mesmo conjunto de dados (bióticos e abióticos). Cada modelo gerado é comparado, célula a célula, com outro. Somando-se os modelos, é feita nova comparação, novamente célula a célula, para se calcular o quanto um modelo único, ou um modelo resultante de soma, é distante do outro.

\section{Resultados}

Na tabela 1 são apresentadas as 17 espécies lenhosas utilizadas na modelagem, os números de treino e as porcentagens de acerto para cada espécie.

Teste de convergência do algoritmo - Byrsonima intermedia A. Juss. foi escolhida por representar o melhor conjunto de dados disponível, dentre as 17 espécies analisadas, tanto em relação ao número de pontos de amostragem como na porcentagem de acerto (tabela 1). O resultado desta comparação (tabela 2) mostra que os modelos resultantes para os cinco conjuntos de dados, gerados a partir da soma de 100 modelos, são bastante semelhantes (figura 2).

Além disso, considerou-se mais eficiente a utilização de 100 modelos, uma vez que rodar mais modelos exigiria um tempo de processamento bem maior sem que isso trouxesse um resultado significativamente melhor para os modelos somados resultantes.

Especificidade ambiental dos pontos de amostragem no resultado da modelagem - Na figura 3 é apresentado o resultado da modelagem obtida nos dois experimentos. A modelagem utilizando apenas localidades de cerrado (experimento A) mostrou uma porcentagem de acerto maior que a modelagem realizada a partir de pontos com fisionomias de outros biomas (experimento B), apesar do maior número de pontos deste segundo experimento (figura 3; tabela 1). Para um mesmo número de pontos de treino e teste, o experimento $\mathrm{A}$ foi sempre o que apresentou maior porcentagem de acerto (figura 3), para todas as espécies. Essas diferenças não são explicadas pelo acaso (tabela 2 ) e representam um efeito consistente da escolha dos pontos sobre a eficácia do modelo.

$\mathrm{Na}$ figura 4 é apresentado o mapa potencial da distribuição de cerrado no Estado de São Paulo e na figura 5 são apresentados os modelos gerados para cada espécie analisada.

\section{Discussão}

Esses resultados mostram que a eficácia do modelo aumenta se forem utilizadas localidades em que o bioma é único, sem fisionomias de biomas vizinhos. Quando as espécies têm distribuição mais ampla, ocorrendo também em bordas de fragmentos pertencentes a outras formações vegetais, essas ocorrências podem diminuir a porcentagem de acerto do modelo resultante. Isso provavelmente deve estar relacionado ao número desigual de pontos provenientes de diferentes biomas. No caso do experimento A foram incluídas 69 áreas,

Tabela 2. Análise de covariância para a porcentagem de acerto nos experimentos A e B e o efeito do número de pontos de teste $(g l$ trat $=$ graus de liberdade do tratamento; $g l$ erro $=$ graus de liberdade do erro; $P=$ probabilidade do resultado ser dado ao acaso).

Table 2. Covariance analysis for the percentage of correct predictions in experiments A and B and the effect of the number of test points $(g l$ trat $=$ degrees of freedom of the treatment; $g l$ error $=$ degrees of freedom of the error; $P=$ probability of the result being obtained by chance).

\begin{tabular}{lcccc}
\hline Comparação & $F$ & $g l$ trat & $g l$ erro & $P$ \\
\hline Diferença de inclinação & 0,566 & 1 & 30 & 0,458 \\
Efeito do tipo de experimento & 40,783 & 1 & 30 & $<0,001$ \\
Efeito do número de amostras & 92,257 & 1 & 30 & $<0,001$ \\
\hline
\end{tabular}




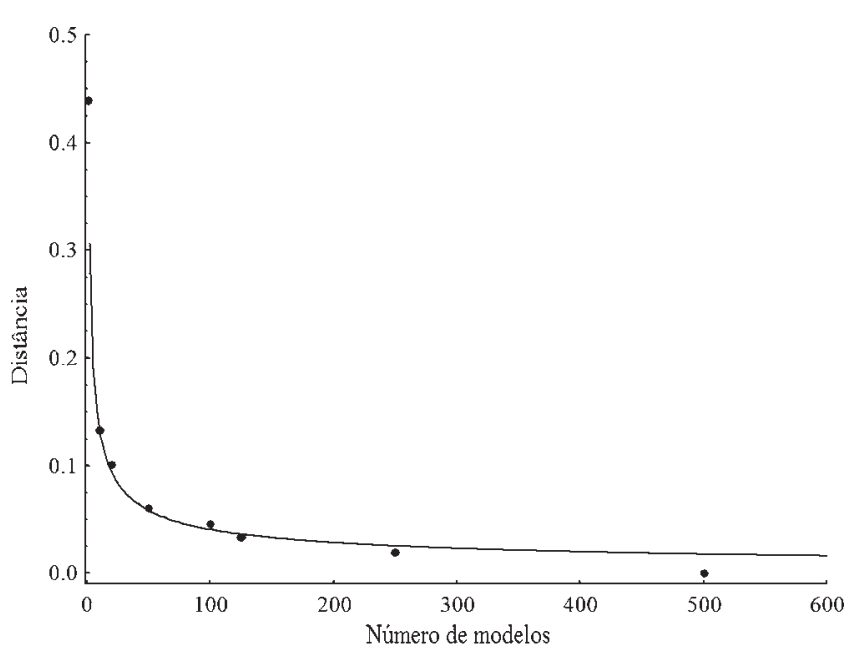

Figura 2. Média das distâncias entre modelos baseados na ocorrência de Byrsonima intermedia A. Juss., em função do número de modelos somados. A linha representa o ajuste produzido por técnica de ajuste não-linear a um modelo de função potência. A Equação da curva é $D=0,441 N^{-0,517}$; onde $D$ é a distância e $N$ o número de modelos somados.

Figure 2. Distances average between the models of Byrsonima intermedia A. Juss., summarized by the number of models. The line represents the non-linear adjustment using potency function. The curve equation is $D=0,441 N^{-0,517}$, where $D$ is the distance and $N$ is the number of summarized models.

sendo 66 áreas de cerrado e três áreas contendo fisionomias tanto de cerrado, como de ecótono e floresta estacional. No experimento B foram incluídas todas as 86 áreas, sendo 66 áreas com vegetação típica de cerrado, 11 áreas com vegetação ecotonal, seis áreas de floresta estacional semidecidual e três áreas contendo as fisionomias de cerrado, ecótono e floresta estacional. Ou seja, no segundo caso (experimento B) o algoritmo tem poucos pontos de amostragem de floresta estacional e ecótono em comparação com as áreas de cerrado. Portanto, é de se esperar uma capacidade mais baixa na porcentagem de acerto do modelo. Portanto, a eficácia do modelo em predizer a ocorrência de espécies do cerrado é maior se forem utilizados apenas pontos de amostragem com fisionomias de cerrado, excluindo-se áreas de transição.

Por outro lado, se o que se busca é um modelo de distribuição mais inclusivo de determinada espécie, com o objetivo de definir os locais onde a espécie tem condições de sobreviver, então é aconselhável utilizar todos os registros disponíveis de ocorrência daquela espécie, dentro da área de estudo.

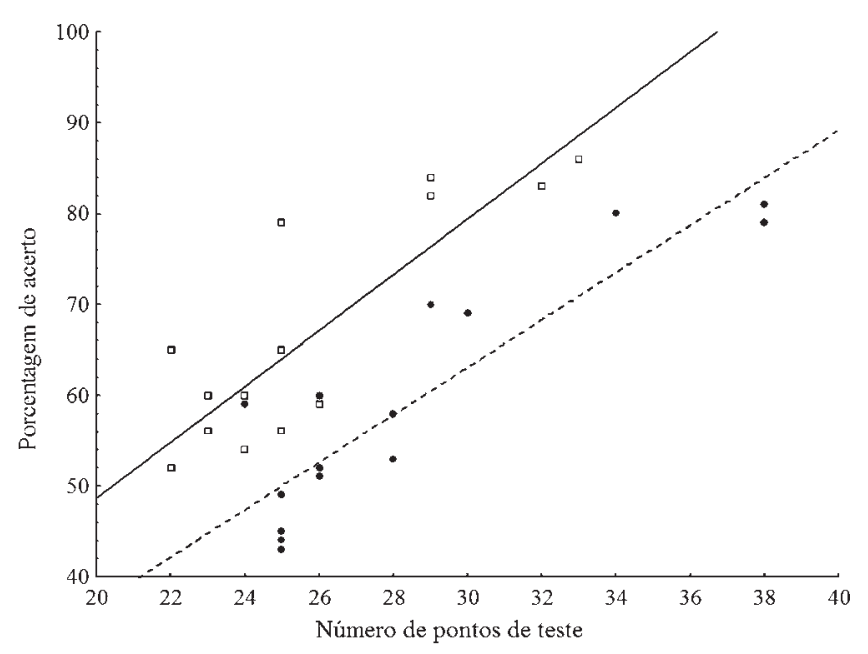

Figura 3. Comparação entre a porcentagem de acerto de dois conjuntos de pontos de treino para a modelagem do bioma cerrado em São Paulo. $-\square-$ - $=$ Experimento A: localidades de amostragem contendo apenas áreas de cerrado. --•-- = Experimento B: localidades de cerrado, floresta estacional e vegetação de transição entre as duas (região de ecótono cerrado/floresta).

Figure 3. Comparison between percentages of correct predictions of two sets of training points used in modeling the "cerrado" biome in São Paulo. - $-\square-=$ Experiment A: the sampled localities only contain cerrado areas. -- $\bullet--=$ Experiment B: includes localities of the "cerrado", stational forests, and vegetation of the transition area (ecotone area "cerrado"/forest).

Distribuição potencial do bioma cerrado no Estado de São Paulo - A distribuição potencial do cerrado gerada pela modelagem resultou em uma área bem maior que a área mapeada pelo IBGE (1988) (figuras 1 e 4). Isso também era verdadeiro no início dos anos 1970, como sugere a revisão de Eiten (1972). A subestimativa da distribuição do cerrado foi particularmente severa em relação à região Oeste do Estado. $\mathrm{O}$ autor cita especificamente a presença de importantes áreas de cerrado na região de Paraguaçu Paulista e Rancharia (Sudoeste do Estado), no Nordeste de São José do Rio Preto, na região do Rio Grande (Norte do Estado), várias áreas de cerradão no Noroeste do Estado e também muitas áreas menores dispersas na região Oeste que não eram incorporadas nos mapeamentos de cerrado. Ainda considerando o Estado de São Paulo, para Eiten (1972) o melhor mapeamento do cerrado foi feito por Borgonovi \& Chiarini (1965), mas mesmo esses autores deixaram de incluir algumas áreas, novamente as áreas do Nordeste de São José do Rio Preto e uma área grande em São Carlos. 
Tanto o modelo gerado (figura 4A), como o recente mapeamento do Instituto Florestal (figura 4B), corroboram a idéia de subamostragem, mostrando uma área de cerrado potencialmente maior. Os fragmentos atuais de cerrado podem ser encontrados tanto nas áreas mapeadas como floresta estacional semidecidual como nas áreas de vegetação ecotonal, do mapa do IBGE (1988) (figura 1).

$\mathrm{Na}$ prática é bastante difícil estabelecer os limites entre o cerrado e a floresta estacional no Estado. A região ecotonal é bastante descontínua, formando um mosaico de vegetação, ocorrendo fragmentos de cerrado e fragmentos de floresta estacional e extensas áreas de vegetação de transição. Segundo Oliveira-Filho \& Ratter (2002) onde os solos são mais férteis, a vegetação climax é definitivamente floresta mesofítica (incluindo florestas decíduas e semidecíduas).

Esses resultados sugerem que para a escala geográfica analisada, dados climáticos, topográficos e dados de solos (mesmo categorizados) geram um bom modelo de distribuição do bioma cerrado, mas ainda assim os modelos gerados apresentam problemas, ou seja, o modelo não garante sua precisão em todas as situações. Porém, é de se esperar que com a inclusão de dados contínuos de solos (riqueza, granulometria, capacidade de absorção e manutenção da água, etc.) na modelagem, a distinção entre a distribuição do cerrado e da floresta estacional semidecidual possa ser melhor mapeada pelas técnicas utilizadas.

Em relação às 17 espécies modeladas, com base nos resultados obtidos (figura 5), verifica-se que há diferentes especificidades na distribuição de cada espécie. Esse resultado corrobora aqueles obtidos por Durigan et al. (2003b), que encontraram padrões fitogeográficos distintos para o Estado. Aparentemente, algumas espécies não são sensíveis a variações ambientais dentro do Estado (Brosimum gaudichaudii Trécul., Anadenanthera falcata (Benth.) Speg., Byrsonima intermedia A. Juss., Duguetia furfuracea (A. St.-Hil.) Benth. \& Hook f.), enquanto outras são restritas a determinadas regiões (Aegiphila lhotskiana Cham., Annona dioica St.-Hil., Campomanesia adamantium (Cambess.) O. Berg., Miconia stenostachya DC. e Caryocar brasiliense Cambess.).

A junção de todas as 17 espécies em um único modelo resulta em um mapa que é, provavelmente, muito próximo da distribuição original do cerrado em São Paulo. Aplicações dos modelos resultantes - O mapa potencial da distribuição de cerrado no Estado de São Paulo (figura 4) pode servir de base para o desenvolvimento de projetos conservacionistas, como, por exemplo, para a formação de corredores de vegetação ou áreas de conservação ex situ, ou ainda na escolha de áreas prioritárias para a conservação deste bioma.

Diferentes espécies apresentam diferentes padrões de distribuição no Estado, evidenciando as diferentes necessidades ambientais e diferentes fatores limitantes para a distribuição dessas espécies. Para as espécies analisadas, os mapas de distribuição potencial de espécies (figura 5) podem servir de base para definir quais as espécies mais indicadas para recuperação de
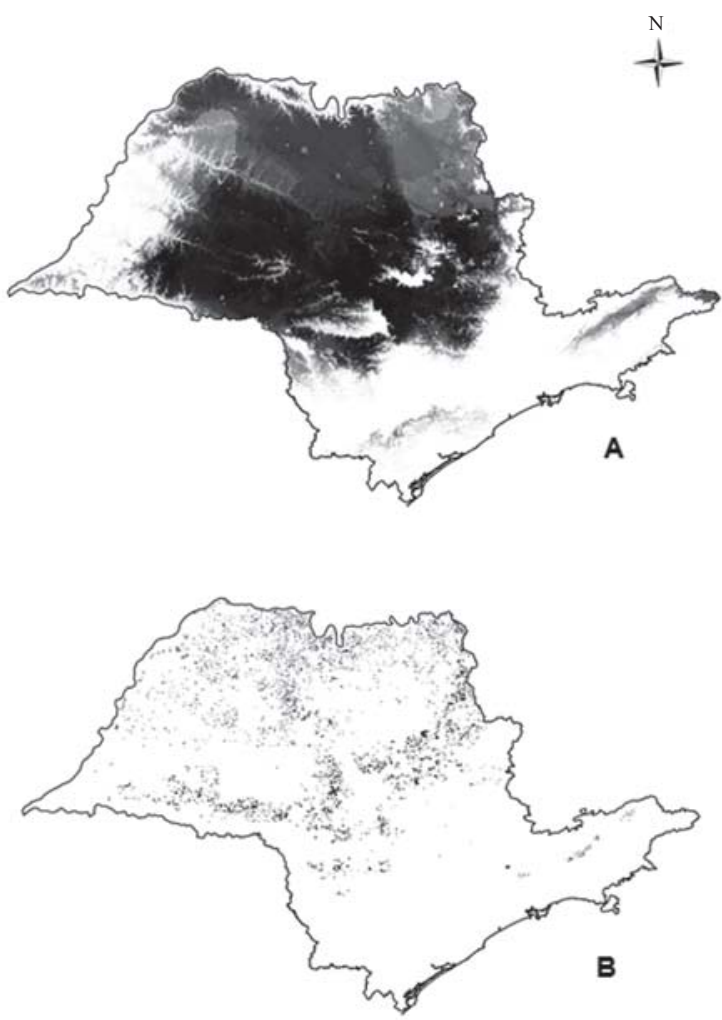

Figura 4. A. Distribuição potencial do bioma cerrado para o Estado de São Paulo, obtida a partir da soma dos 10 melhores modelos (entre os 100 gerados) para 17 espécies de cerrado, utilizando o algoritmo genético GARP. O gradiente cinza representa o número de modelos em ordem crescente, o branco significa zero modelos e o cinza mais escuro representa a área de intersecção dos 10 modelos somados. B: Distribuição dos remanescentes de cerrado no Estado de São Paulo (Kronka et al. 1998).

Figure 4. A. Potential distribution of the "cerrado" biome in São Paulo State summarized by the 10 best subsets for 17 species modeled by GARP. The grey gradient represents the number of models in ascending order. The white zone represents zero models and the dark grey zone represents the intersection area of all 10 models. B. Remainders of natural vegetation in São Paulo State (Kronka et al. 1998). 


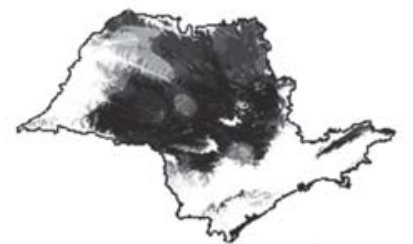

Aegiphila Ihotskiana Cham.

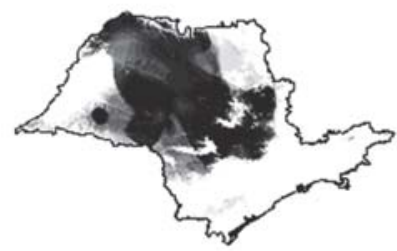

Annona dioica A. St.-Hil.

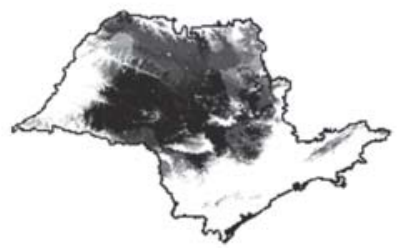

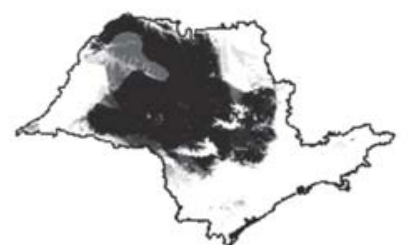

Anadenathera falcata (Benth.) Speg.

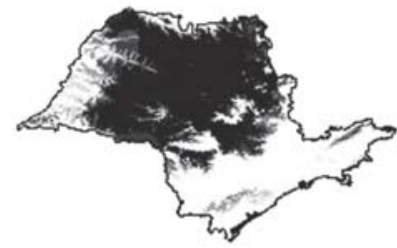

Brosimum gaudichaudii Trecul

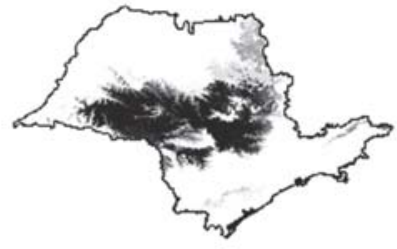

Caryocar brasiliense Cambess.

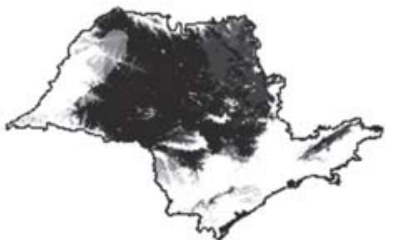

Gochnatia barrosii Cabrera

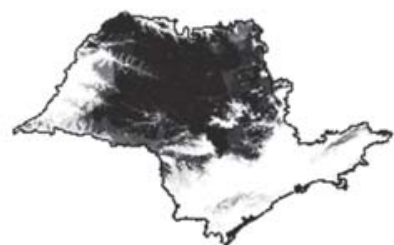

Annona coriacea Mart.

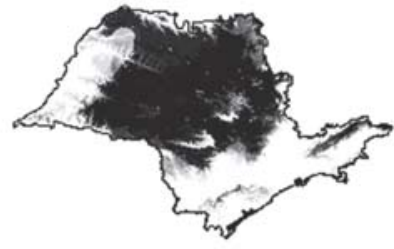

Byrsonima intermedia A. Juss.

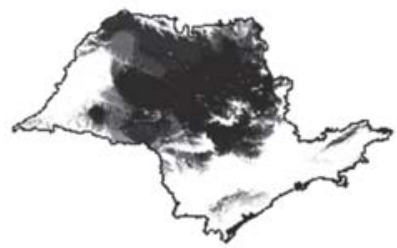

Dimorphandra mollis Benth.

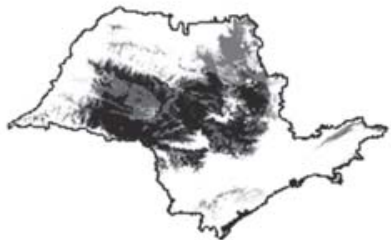

Miconia stenostachya DC.

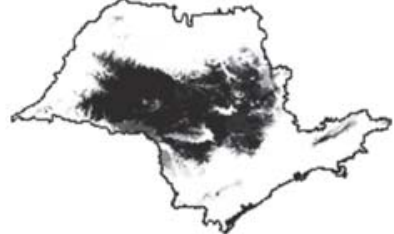

Ouratea spectabilis (Mart.) Engl.

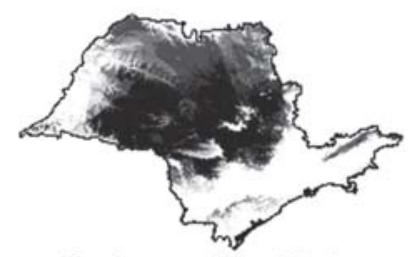

Qualea grandiflora Mart.

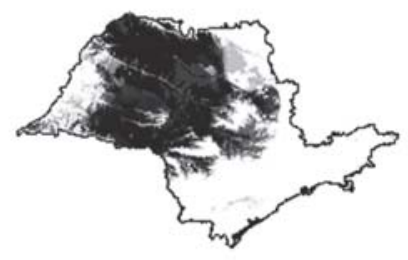

Schefflera vinosa (Cham. \& Schltdl.) Frodim

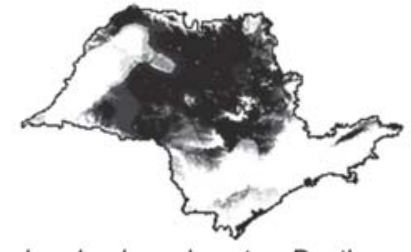

Stryphnodendron obovatum Benth.

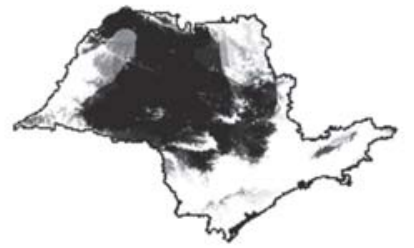

Tocoyena formosa (Cham. \& Schltdl.) K. Schum.

Figura 5. Distribuição potencial de espécies de cerrado no Estado de São Paulo, obtida a partir da soma dos 10 melhores modelos entre os 100 gerados pelo GARP. O gradiente cinza representa o número de modelos em ordem crescente, o branco significa zero modelos e o cinza mais escuro representa a área de intersecção dos 10 modelos somados.

Figure 5. Potential distribution of species of the "cerrado" biome in São Paulo State summarized by the 10 best subsets of the 100 generate by GARP. The grey gradient represents the number of models in ascending order. The white zone represents zero models and the dark grey zone represents the intersection area of all 10 best models. 
áreas degradadas dentro do domínio dos cerrados no Estado.

O uso de modelagem pode auxiliar no entendimento dos padrões de distribuição de um bioma ou ecossistema em uma análise regional, como é o caso da distribuição de cerrado no Estado de São Paulo. Esta área foi sistematicamente subestimada no passado. Segundo a modelagem, as áreas potenciais de ocorrência de espécies de cerrado são mais amplas do que as áreas apontadas pelos mapas existentes.

Agradecimentos - Os autores agradecem ao Prof. Dr. Andrew Townsend Peterson e Ricardo Scachetti, por terem apresentado a modelagem como ferramenta para tratar dados levantados no campo e pelo valioso auxílio e orientação no desenvolvimento da modelagem. Sidnei de Souza, Paulo de Marco Júnior e Mauro Enrique de Souza Muñoz, pelo auxílio no desenvolvimento das etapas de modelagem deste trabalho e na análise dos dados. Dora Ann Lange Canhos e Ingrid Koch, pela leitura crítica do manuscrito. Geraldo Antônio Daher Corrêa Franco e Edivaldo Furlan pelo auxílio na identificação e levantando dos dados. À FAPESP, que, através do Programa Biota, financiou o projeto "Viabilidade de conservação dos fragmentos de cerrado do Estado de São Paulo", no qual foram levantadas as espécies arbóreas de cerrado no Estado de São Paulo utilizadas neste trabalho e o projeto Sistema de informação distribuído para coleções biológicas: a integração do Species Analyst e do SinBiota, no qual foram desenvolvidas as técnicas de modelagem.

\section{Referências bibliográficas}

ANDERSON, R.P., LEW, D., \& PETERSON, A.T. 2003. Evaluating predictive models of species' distributions: criteria for selecting optimal models. Ecological Modelling 162:211-232.

BEHLING, H. 1998. Late Quaternary vegetational and climatic changes in Brazil. Review of Palaeobotany and Palynology 99:143-156.

BEHLING, H. 2003. Late glacial and Holocene vegetation, climate and fire history inferred from Lagoa Nova in the southeastern Brazilian lowland. Vegetation History and Archaeobotany 12:263-270.

BIOTA. 1999. Programa Biota/Fapesp. http:// www.biota.org.br/ (acessado em 09/10/2006).

BORGONOVI, M. \& CHIARINI, J.V. 1965. Cobertura vegetal do Estado de São Paulo. I. Levantamento por fotointerpretação das áreas cobertas com cerrado, cerradão e campo em 1962. Bragantia 24:159-172.

DURIGAN, G, RATTER, J.A., BRIDGEWATER, S., SIQUEIRA, M.F. \& FRANCO, G.A.D.C. 2003a. Padrões fitogeográficos do cerrado paulista sob uma perspectiva regional. Hoehnea 30:39-51.
DURIGAN, G., SIQUEIRA, M.F., FRANCO, G.A.D.C., BRIDGEWATER, S. \& RATTER, J.A. 2003b. The vegetation of priority areas for cerrado conservation in São Paulo State, Brazil. Edinburgh Journal of Botany 60:217-241.

DURIGAN G., BAITELO, J.B., FRANCO, G.A.D.C. \& SIQUEIRA, M.F. 2004. Plantas do cerrado paulista. Imagens de uma paisagem ameaçada. Instituto Florestal, Secretaria do Meio Ambiente do Estado de São Paulo. Japan International Cooperation Agency - JICA. Páginas e Letras Editora e Gráfica, São Paulo.

EITEN, G. 1970. A vegetação do Estado de São Paulo. Boletim do Instituto de Botânica 7:1-77.

EITEN, G. 1972. The cerrado vegetation of Brazil. The Botanical Review 38:201-341.

EROS. 1996. HYDRO1k - Elevation derivative database. http:// edc.usgs.gov/products/elevation/gtopo30/hydro/ index.html. (acessado em 09/10/2006).

HIJMANS, R.J., CAMERON, S.E., PARRA, J.L., JONES, P.G. \& JARVIS, A. 2005. Very high resolution interpolated climate surfaces for global land areas. International Journal of Climatology 25: 1965-1978.

IBAMA. 1993. Decreto $\mathrm{n}^{\circ}$ 750, de 10 de fevereiro de 1993. http://www.ibama.gov.br/flora/decretos/750_93.pdf (acessado em 09/10/2006).

IBGE. 1988. Vegetation Map of Brazil. Instituto Brasileiro de Desenvolvimento Florestal. Secretaria de Planejamento e Coordenação da Presidência da Republica. Fundação Instituto Brasileiro de Geografia e Estatística - IBGE. http://www.whrc.org/southamerica/LBAData/ Braz_Veg_IBGE.htm. (acessado em 09/10/2006).

KRONKA, F.J.N., NALON, M.A., MATSUKUMA, C.K., PAVÃO, M., GUILLAUMON, J.R., CAVALLI, A.C., GIANNOTTI, E., IWANE, M.S.S., LIMA, L.M.P.R., MONTES, J., DEL-CALI, I.H. \& HAACK, P.G. 1998. Áreas de domínio de cerrado no Estado de São Paulo. Secretaria de Estado do Meio Ambiente, Instituto Florestal, São Paulo.

LEDRU, M.P. 2002. Late Quaternary history and evolution of the cerrados as revealed by palynological records. In The cerrados of Brazil - Ecology and natural history of neotropical savanna. (P.S. Oliveira \& R.J. Marquis, orgs.). Columbia University Press, New York, p.33-50.

LEDRU, M.P., SALGADO-LABOURIAU, M.L. \& LORSCHEITTER, M.L. 1998. Vegetation dynamics in southern and central Brazil during the last 10,000 yr B.P. Review of Palaeobotany and Palynology 99:131-142.

MOTTA, P.E.F., CURI, N. \& FRANZMEIER, D.P. 2002. Relation of soils and geomorphic surfaces in the Brazilian cerrado. In The cerrados of Brazil - Ecology and natural history of neotropical savanna. (P.S. Oliveira \& R.J. Marquis, orgs.). Columbia University Press, New York, p.13-32.

OLIVEIRA, J.B., CAMARGO, M.N., ROSSI, M. \& CALDERANO FILHO, B. 1999. Mapa pedológico do Estado de São Paulo: legenda expandida. Instituto Agronômico/Embrapa Solos. Campinas. 
OLIVEIRA FILHO, A.T., \& RATTER, J. A. 2002. Vegetation physiognomies and woddy flora of the cerrado Biome. In The cerrados of Brazil - Ecology and natural history of neotropical savanna. (P.S. Oliveira \& R. J. Marquis, orgs) Columbia University Press, New York, p.91-120.

RATTER, J.A., \& DARGIE, T.C.D. 1992. An analysis of the floristic composition of 26 cerrado areas in Brazil. Edinburg Journal of Botany 49:235-250.

RATTER, J.A., BRIDGEWATER, S., RIBEIRO, J.F., DIAS, T.A.B. \& SILVA, M.R. 2000. Distribuição das espécies lenhosas da fitofisionomia cerrado sentido restrito nos estados compreendidos no bioma cerrado. Boletim do Herbário Ezechias Paulo Heringer 5:5-43.

SALGADO-LABOURIAU, M.L. 1998. Late Quaternary palaeoclimate in the savannas of South America. Journal of Quaternary Science 12:371-379.

SALGADO-LABOURIAU, M.L., CASSETI, V., FERRAZVICENTINI, K.R., MARTIN, L., SOUBIÈS, F., SUGUIO, K. \& TURCQ, B. 1997. Late Quaternary vegetational and climatic changes in cerrado and palm swamp from Central Brazil. Palaeogeography, Palaeoclimatology, Palaeoecology 128:215-226.
SALGADO-LABOURIAU, M. L., BARBIERI, M., FERRAZVICENTINI, K.R. \& PARIZII, M.G. 1998. A dry climatic event during the late Quaternary of tropical Brazil. Review of Palaeobotany and Palynology 99:115-129.

SERRA FILHO, R., CAVALLI, A.C., GUILLAUMON, J.R., CHIARINI, J.V., NOGUEIRA, F.P., IVANCKO, C.M.A.M., BARBIERI, J.L., DONIZELI, P.L., COELHO, A.G.S. \& BITTENCOURT, I. 1974. Levantamento da cobertura natural e do reflorestamento no Estado de São Paulo. Boletim Técnico do Instituto Florestal 11:1-56.

SINBIOTA. 1999. Sistema de informação ambiental do Biota. http://sinbiota.cria.org.br/ (acessado em 09/10/2006).

SMA-SP. 1997. Cerrado: bases para a conservação e uso sustentável das áreas de cerrado do Estado de São Paulo. Secretaria de Estado do Meio Ambiente. São Paulo.

STOCKWELL, D.R.B. \& PETERSON, A. T. 2002. Effects of sample size on accuracy of species distribution models. Ecological Modelling 148:1-13.

VARGAS, M.A.T. \& HUNGRIA, M. 1997. Biologia dos solos dos cerrados. Embrapa-CPAC, Planaltina.

VICTOR, M.A.M. 1975. A devastação florestal. Sociedade Brasileira de Silvicultura. São Paulo. 